\title{
Association of X-ray Absorptiometry Body Composition Measurements with Basic Anthropometrics and Mortality Hazard
}

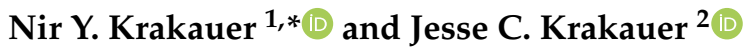 \\ 1 Department of Civil Engineering, City College of New York, New York, NY 10031, USA \\ 2 Associated Physicians/Endocrinology, Berkley, MI 48072, USA; jckrakauer@gmail.com \\ * Correspondence: nkrakauer@ccny.cuny.edu
}

Citation: Krakauer, N.Y.; Krakauer, J.C. Association of X-ray

Absorptiometry Body Composition Measurements with Basic Anthropometrics and Mortality Hazard. Int. J. Environ. Res. Public Health 2021, 18, 7927. https:// doi.org/10.3390/ijerph18157927

Academic Editor: Shane Norris

Received: 14 June 2021

Accepted: 25 July 2021

Published: 27 July 202

Publisher's Note: MDPI stays neutral with regard to jurisdictional claims in published maps and institutional affiliations.

Copyright: (c) 2021 by the authors. Licensee MDPI, Basel, Switzerland This article is an open access article distributed under the terms and conditions of the Creative Commons Attribution (CC BY) license (https:// creativecommons.org/licenses/by/ $4.0 /)$

\begin{abstract}
Dual-energy X-ray absorptiometry (DEXA) is a non-invasive imaging modality that can estimate whole-body and regional composition in terms of fat, lean, and bone mass. We examined the ability of DEXA body composition measures (whole-body, trunk, and limb fat mass and fat-free mass) to predict mortality in conjunction with basic body measures (anthropometrics), expressed using body mass index (BMI) and a body shape index (ABSI). We used data from the 1999-2006 United States National Health and Nutrition Examination Survey (NHANES), with mortality follow-up to 2015. We found that all DEXA-measured masses were highly correlated with each other and with ABSI and that adjustment for BMI and ABSI reduced these dependencies. Whole-body composition did not substantially improve mortality prediction compared to basic anthropometrics alone, but regional composition did, with high trunk fat-free mass and low limb fat-free mass both associated with elevated mortality risk. These findings illustrate how DEXA body composition could guide health assessment in conjunction with the more widely employed simple anthropometrics.
\end{abstract}

Keywords: dual-energy X-ray absorptiometry; obesity; sarcopenia; risk assessment; anthropometry; body shape

\section{Introduction}

The basic anthropometrics of height, weight, and waist circumference (WC), and the derived quantities body mass index (BMI, weight adjusted for height) and a body shape index (ABSI, WC adjusted for weight and height) are robust predictors of mortality hazard as well as many health conditions and forms of morbidity [1,2]. However, it is recognized that these basic body measures do not directly measure body composition-in particular, the amount, location, and type of fat and muscle tissue [3-6]. A number of imaging methods are available that provide such information, although all are much less widely used than basic anthropometrics, and their benefits for prognosis are less well quantified [7].

Of these imaging modalities, dual-energy X-ray absorptiometry (DEXA) scanning is considered a reference method for the assessment of human body composition, due to its relative non-invasiveness, good discrimination ability, and low cost compared to other techniques [8,9]. Originally employed only to monitor bone mass and density for fracture risk assessment, DEXA was established in the 1990s to also distinguish between fat and lean tissue in the different body regions [10,11]. DEXA measurements, along with other clinical and laboratory examinations, were conducted for the 1999-2006 cohorts of the United States National Health and Nutrition Examination Survey (NHANES), comprising over 10,000 individuals selected to be broadly representative of the national population. These NHANES measurements were used to develop age-specific body composition reference values $[12,13]$.

With the availability of follow-up data on mortality of NHANES subjects, a number of studies have also assessed how body composition correlated with mortality. These studies focused on whether there was a contrast in the mortality association of fat mass versus fat-free mass [14-16], with trunk fat being possibly particularly associated with adverse 
cardiometabolic outcomes linked to abdominal obesity, and also whether a protective effect could be seen of high limb lean tissue mass (which is primarily muscle) [17-20]. However, while these studies mostly adjusted for BMI, they did not consider the correlation of DEXA body composition and its association with mortality risk with ABSI, which in some populations is a more powerful mortality predictor than BMI and which is correlated with fat percentage and with muscle mass [21-24].

The work presented here analyzes NHANES data to determine (a) the association between simple anthropometrics (BMI and ABSI) and DEXA-based whole-body and regional (limb, trunk) composition (fat and lean mass), and (b) the association between the DEXA-based measures and mortality hazard and the extent to which they improve hazard assessment over using only simple anthropometrics. Our goal is to contribute to defining the value of DEXA body composition scans for prognosis and to explore how these scans could be used in conjunction with the more widely employed simple anthropometrics.

\section{Methods}

\subsection{NHANES}

NHANES has been sampling the civilian non-institutionalized USA population since 1971 using a cluster approach. Some groups of public health interest (children, the elderly, black and Mexican-American people) were deliberately oversampled. We analyzed the 1999-2000, 2001-2003, 2003-2004, and 2005-2006 NHANES cohorts, for which body composition parameters were measured using DEXA. We considered all adults (age 18 and over) with complete basic anthropometric and DEXA measurements and mortality follow-up. Mortality outcomes for adult subjects were available from the National Center for Health Statistics through 2015 (9-16 years of follow-up, median: 12.5 years).

DEXA scans in NHANES used the QDR 4500A fan beam densitometer (Hologic, Inc., Bedford, MA, USA) and Hologic Discovery software version 12.1. People who were taller than $196 \mathrm{~cm}$, heavier than $136 \mathrm{~kg}$, or pregnant were not scanned. We also excluded individuals with any missing data due to limb amputations, prosthetics or implants, or other factors that affected scan quality (which were particularly common at higher BMI levels and older ages) [25]. Therefore, the cohort studied cannot be taken as representative of the entire adult population but only of those whose whole-body DEXA scans would yield complete and valid results.

The protocol for NHANES has been approved by the National Center for Health Statistics Research Ethics Review Board as consistent with the Declaration of Helsinki. Ethics approval was not needed for the current study because only anonymized, public-use data (https:/ / www.cdc.gov/nchs/nhanes/index.htm, accessed on 1 June 2021) is employed.

\subsection{Indices and Standardization}

Anthropometric indices were calculated as follows [26,27]:

$$
\begin{gathered}
\mathrm{BMI} \equiv \mathrm{W} \cdot \mathrm{H}^{-2}, \\
\mathrm{ABSI} \equiv \mathrm{WC} \cdot \mathrm{H}^{5 / 6} \cdot \mathrm{W}^{-2 / 3},
\end{gathered}
$$

where $\mathrm{W}$ designates weight, $\mathrm{H}$ height, and $\mathrm{WC}$ waist circumference.

Similar to BMI, the mass estimated by DEXA is divided by the square of measured height to construct body composition indices [28,29]. Thus, fat mass index (FMI) is total fat mass divided by $H^{2}$, fat-free mass index (FFMI) is total non-fat mass divided by $H^{2}$, and similarly for the trunk and limbs (limb fat mass index LFMI, limb fat-free mass index LFFMI, trunk fat mass index TFMI, trunk fat-free mass index TFFMI).

The anthropometric or body composition index values are converted to $\mathrm{Z}$ scores by subtracting the smoothed age and sex-specific mean and dividing by the standard deviation [23], thus following the general formula:

$$
\text { Z-score } \equiv \frac{\text { value }- \text { mean }}{\text { std. dev. }} .
$$


To better understand the interrelation of basic anthropometrics and body composition, correlations were calculated between the $\mathrm{Z}$ scores of the different indices listed above. ABSI and BMI are defined so that the correlation between them is close to zero [27]. Following the same approach, allometrically adjusted forms of the body composition indices can be found that have close to zero correlation with BMI and ABSI by first finding the least-squares coefficients for the linear regression

$$
\log \text { index } \sim \text { intercept }+\alpha \log \mathrm{H}+\beta, \log \mathrm{BMI}+\gamma \log \mathrm{ABSI}+\sigma \operatorname{sex}
$$

where sex is set to 1 for females and 0 for males, and then adjusting for anthropometrics accordingly:

$$
\text { adjusted-index }=\frac{\text { index }}{\left(\frac{\mathrm{H}}{\langle\mathrm{H}\rangle}\right)^{\alpha}\left(\frac{\mathrm{BMI}}{\langle\mathrm{BMI}\rangle}\right)^{\beta}\left(\frac{\mathrm{ABSI}}{\langle\mathrm{ABSI}\rangle}\right)^{\gamma}} .
$$

Here, $\langle\mathrm{H}\rangle=166 \mathrm{~cm},\langle\mathrm{BMI}\rangle=26 \mathrm{~kg} \mathrm{~m}^{-2}$, and $\langle\mathrm{ABSI}\rangle=0.0803 \mathrm{~m}^{11 / 6} \mathrm{~kg}^{-2 / 3}$ are median values from NHANES III, used to normalize the body composition measures to a standard height, weight, and WC. This makes the adjusted indices approximately independent of BMI and ABSI as well as height [23]. The adjusted indices are then transformed to $\mathrm{Z}$ scores, as before, which standardizes variability due to age and sex.

\subsection{Statistical Modeling of Association with Mortality}

Cox proportional hazard modeling [30] was used to assess the impact of body composition indices, with or without anthropometrics on death rate (mortality hazard) over the follow-up period, helping determine which aspects of body composition could be related to mortality risk and what forms these relationships took. All mortality hazard models were compared to a baseline model, which included only age (used as the timescale in the Cox model), sex (male/female), and race (black/nonblack) as predictors [23,27,31].

As in previous studies $[23,32,33]$, the main measure of relative model performance was the Akaike information criterion (AIC), which was expressed as a difference from the baseline model:

$$
\Delta_{\text {model } m} \equiv \mathrm{AIC}_{\text {baseline }}-\mathrm{AIC}_{\text {model } m} .
$$

Lower AIC (higher $\Delta$ ) indicates models that perform better as mortality predictors for the sampled population. The expected likelihood of each model given the NHANES data is proportional to $e^{\Delta / 2}$, so that a difference of 6 is the approximate threshold for significance at the $95 \%$ confidence level (since $e^{-6 / 2} \approx 5 \%$ ) [34].

Two additional measures of model mortality-prediction performance were also computed and considered. One was the $R^{2}$ statistic, defined as a proportion of variation in mortality explained by the predictors of each model, so that higher $R^{2}$ suggests a model with greater explanatory power [35]. The other measure computed was concordance (C), defined as the fraction of pairs of individuals in the sample for which the one modeled to be at greater risk actually died sooner [36]. Concordance ranges from 0 to 1, with 0.5 the expected value for models with no skill and higher values indicating models that are more skillful at explaining variation in survival. Concordance is a generalization of the area under the receiver operating characteristic curve (AUC), which can also range from 0 to 1 and which can be interpreted as the fraction of pairs of individuals where the one who died had the higher predictor value. AUC in its basic form is not a suitable measure here because it does not straightforwardly account for the time to death and because it assumes the hazard to be a monotone function of the predictor, which we expected to generally not be the case $[37,38]$.

The anthropometric and body composition $\mathrm{Z}$ scores, used as predictors, could enter into the mortality hazard models either linearly or nonlinearly. For a linear predictor, each unit increase in its $\mathrm{Z}$ score would increase or decrease the logarithm of mortality hazard by a constant amount. For a nonlinear predictor, the logarithm of mortality hazard would be shifted by an arbitrary smooth function of the $\mathrm{Z}$ score, expressed using a penalized spline 
basis, with corrected AIC being used to choose the function's complexity $[27,36,39]$. Of the anthropometric and body composition predictors considered here, ABSI was entered into each model as a linear predictor, since it has previously been shown that the logarithm of mortality hazard has a near-linear dependence on the ABSI Z score [2,27], while BMI and the body composition indices were entered as nonlinear predictors to allow for the possibility of, for example, U-shaped associations with mortality risk.

All analyses were conducted in the R programming language, version 4.0.4 [40]. The implementation of Cox proportional hazard modeling in the coxph function of the survival package, version 3.2.7, was used [36,41].

\section{Results}

\subsection{Sample Characteristics and Correlations}

Basic demographics of the NHANES sample are given in Table 1. The average age is a slight underestimate because, to ensure privacy, those 85 years old and over were all listed as 85 in the released NHANES data and formed $1.5 \%$ of the sample. Adjusting for $\mathrm{H}, \mathrm{W}$, WC reduced the variance of body composition measures substantially, with the limb fat mass remaining with the highest coefficient of variation.

Table 1. NHANES 1999-2006 sample characteristics.

\begin{tabular}{|c|c|c|}
\hline & Valid DEXA Scans & All Adults \\
\hline Number & 14,064 & 19,959 \\
\hline Deaths & 2140 & 3478 \\
\hline$\%$ female & 48 & 52 \\
\hline \multirow[t]{5}{*}{ Ethnicity } & Mexican: 25\% & $24 \%$ \\
\hline & Other Hispanic: 4\% & $4 \%$ \\
\hline & White: $47 \%$ & $47 \%$ \\
\hline & Black: $20 \%$ & $21 \%$ \\
\hline & Other: $4 \%$ & $4 \%$ \\
\hline Age (y) & $43 \pm 19$ & $46 \pm 20$ \\
\hline Height $(\mathrm{cm})$ & $168 \pm 10$ & $167 \pm 10$ \\
\hline Weight (kg) & $76 \pm 17$ & $79 \pm 20$ \\
\hline BMI $\left(\mathrm{kg} \mathrm{m}^{-2}\right)$ & $27.1 \pm 5.1$ & $28.1 \pm 6.3$ \\
\hline WC $(\mathrm{cm})$ & $94 \pm 14$ & $96 \pm 16$ \\
\hline ABSI $\left(10^{-2} \mathrm{~m}^{11 / 6} \mathrm{~kg}^{-2 / 3}\right)$ & $8.04 \pm 0.52$ & $8.10 \pm 0.54$ \\
\hline FMI $\left(\mathrm{kg} \mathrm{m}^{-2}\right)$ & $9.2 \pm 3.8[8.6 \pm 2.0]$ & \\
\hline FFMI $\left(\mathrm{kg} \mathrm{m}^{-2}\right)$ & $18.1 \pm 2.8[17.9 \pm 1.8]$ & \\
\hline TFMI $\left(\mathrm{kg} \mathrm{m}^{-2}\right)$ & $4.5 \pm 2.1[4.1 \pm 0.9]$ & \\
\hline TFFMI $\left(\mathrm{kg} \mathrm{m}^{-2}\right)$ & $8.8 \pm 1.3[8.8 \pm 0.9]$ & \\
\hline LFMI $\left(\mathrm{kg} \mathrm{m}^{-2}\right)$ & $4.3 \pm 1.9[4.1 \pm 1.3]$ & \\
\hline LFFMI $\left(\mathrm{kg} \mathrm{m}^{-2}\right)$ & $8.0 \pm 1.5[7.8 \pm 1.0]$ & \\
\hline
\end{tabular}

Basic demographics and body measurements (mean \pm standard deviation) for adults in the NHANES 1999-2006 cohorts, both for those with valid DEXA scans (studied here) and for the entire cohort. Quantities in square brackets are after adjustment to a standard height, weight, and waist circumference. NHANES = National Health and Nutrition Examination Survey; DEXA = dual-energy X-ray absorptiometry; BMI = body mass index; $\mathrm{WC}=$ waist circumference; $\mathrm{ABSI}=\mathrm{a}$ body shape index; FMI = fat mass index (from DEXA scan); FFMI = fat-free mass index; TFMI = trunk fat mass index; TFFMI = trunk fat-free mass index; LFMI $=$ limb fat mass index; LFFMI $=$ limb fat-free mass index.

All the body composition indices showed very high positive correlations with BMI (ranging from 0.81 for LFFMI to 0.94 for FMI), since, as weight increases, the amount of mass in each compartment also increases, and also had high positive correlations with one another (upper right half of Figure 1). Some of the indices were also correlated with height, suggesting that these masses did not scale exactly with height ${ }^{2}$. Furthermore, ABSI correlated positively with TFMI $(r=0.24)$ and negatively with LFFMI $(r=-0.25)$, confirming that high WC for given height and weight is associated with relatively more 
trunk fat and less limb lean mass. As expected from its derivation based on allometric scaling theory [27], ABSI showed almost zero correlation with BMI $(r=0.02)$.

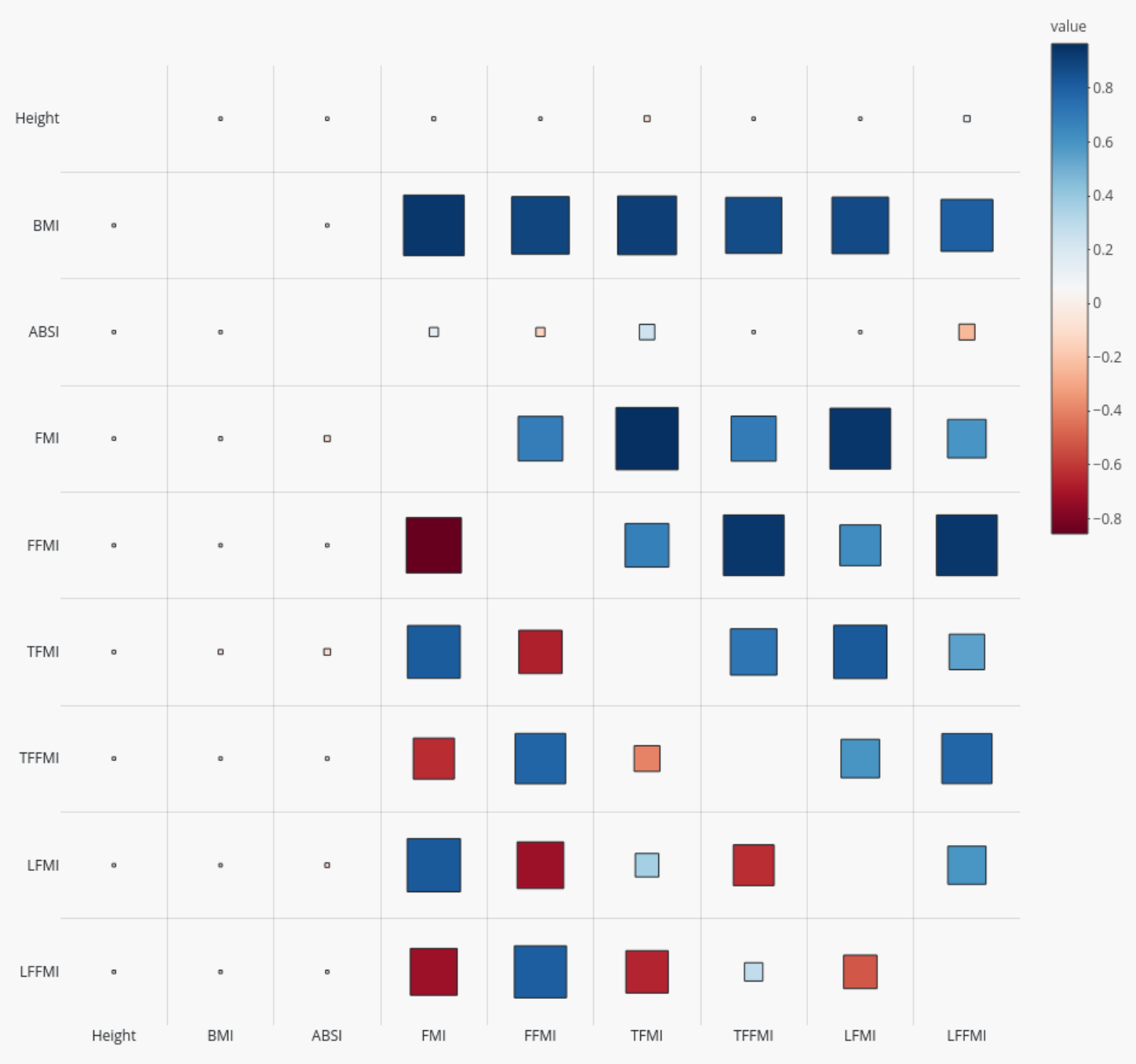

Figure 1. Correlation coefficients for anthropometrics and DEXA-derived body composition indices ( $Z$ scores relative to ageand sex-specific means) among NHANES 1999-2006 adults. Red squares denote positive correlations and blue negative ones; larger size indicates larger correlation magnitude. The upper right half of the correlation matrix shows correlations of the unadjusted indices, while for the lower left half, the body composition indices were adjusted to a standard height, weight, and waist circumference using power law scaling. NHANES = National Health and Nutrition Examination Survey; DEXA = dual-energy X-ray absorptiometry; BMI = body mass index; WC = waist circumference; $\mathrm{ABSI}=\mathrm{a}$ body shape index; FMI = fat mass index; FFMI = fat-free mass index; TFMI = trunk fat mass index; TFFMI = trunk fat-free mass index; LFMI = limb fat mass index; LFFMI = limb fat-free mass index.

After adjusting for anthropometrics, the correlations of body composition indices with BMI were greatly attenuated $(|r|<0.08$; lower left half of Figure 1). After adjustment, the fat-free mass indices were positively correlated with one another and negatively correlated with the fat mass indices, suggesting a differentiation between people with more fat throughout their body at a given height, weight, and waist circumference versus people with more lean tissue.

The regression coefficients in Table $2(\alpha, \beta, \gamma$ in Equation (4)) confirm the correlations seen between body composition indices and anthropometrics. Taller people have relatively 
less fat mass (particularly trunk fat) and more fat-free mass (particularly limb fat-free mass) at a given BMI. The different indices are not proportional to BMI (which would correspond to $\beta=1$ ) but rather show power-law scaling: as BMI increases, fat mass increases much faster than linearly, while lean mass increases slower than linearly. Finally, at a given height and BMI, high ABSI is correlated with relatively more fat, particularly trunk fat, and less $\operatorname{limb}$ fat-free mass. Overall, as indicated by the coefficients of determination $R^{2}$, over $90 \%$ of the variance in DEXA-measured adult whole-body composition indices, and over $85 \%$ of the variance in regional (trunk and limb) composition, is predictable from sex and basic anthropometrics (Table 2).

Table 2. Regression coefficients for body composition indices from DEXA versus simple anthropometric indices.

\begin{tabular}{ccccc}
\hline Index & Height & BMI & ABSI & $\boldsymbol{R}^{\mathbf{2}}$ \\
\hline FMI & -0.362 & 1.830 & 1.246 & 0.930 \\
FFMI & 0.140 & 0.611 & -0.364 & 0.912 \\
TFMI & -0.867 & 2.183 & 2.354 & 0.918 \\
TFFMI & 0.013 & 0.600 & -0.011 & 0.863 \\
LFMI & 0.202 & 1.666 & 0.423 & 0.882 \\
LFFMI & 0.491 & 0.672 & -0.764 & 0.879 \\
\hline
\end{tabular}

Regression coefficients for body composition indices versus the anthropometrics height, BMI, and ABSI (Equation (4)), plus the regression coefficient of determination $R^{2}$. BMI = body mass index; WC = waist circumference; $\mathrm{ABSI}=$ a body shape index; FMI $=$ fat mass index; FFMI $=$ fat-free mass index; TFMI $=$ trunk fat mass index; TFFMI = trunk fat-free mass index; LFMI = limb fat mass index; LFFMI = limb fat-free mass index.

\subsection{Associations with Mortality Hazard}

First, we considered each anthropometric or body composition index as a single mortality predictor added to the baseline model with age, sex, and race. The performance of each of these is shown in Table 3 and Figure 2. ABSI was the best single mortality predictor, with significantly higher $\Delta$ than models with any of the other indices; log mortality hazard rose linearly as ABSI Z score increased (Figure 2b). BMI showed increased risk at below-average values but little sign of increased risk at above-average values (Figure 2a). This is different from earlier studies with NHANES populations, which found a U-shaped association between BMI and mortality, with significantly increased risk also at the high end of the BMI range $[23,27]$. The difference is likely due to people with morbid obesity being underrepresented in the subpopulation with valid DEXA scans, which was studied here.

Table 3. The association of each body measure with mortality hazard.

\begin{tabular}{cccc}
\hline Predictor & $\boldsymbol{\Delta}$ & $\boldsymbol{R}^{\mathbf{2}}$ & $\boldsymbol{C}$ \\
\hline Baseline & 0 & 0.031 & 0.567 \\
BMI & 79.3 & 0.056 & 0.581 \\
ABSI & 115.1 & 0.064 & 0.602 \\
FMI & 72.0 & 0.055 & 0.582 \\
FFMI & 46.8 & 0.047 & 0.585 \\
TFMI & 47.6 & 0.047 & 0.579 \\
TFFMI & 31.1 & 0.043 & 0.583 \\
LFMI & 80.3 & 0.057 & 0.586 \\
LFFMI & 99.7 & 0.061 & 0.598 \\
\hline
\end{tabular}

Results of Cox proportional hazard modeling for mortality risk in NHANES 1999-2006 with anthropometric or DEXA body composition index $\mathrm{z}$ scores taken as predictors. The baseline model only included as predictors age, sex, and race, which the other models also all included. NHANES = National Health and Nutrition Examination Survey; DEXA = dual-energy X-ray absorptiometry; BMI = body mass index; WC = waist circumference; $\mathrm{ABSI}=$ a body shape index; FMI = fat mass index; FFMI = fat-free mass index; $\mathrm{TFMI}=$ trunk fat mass index; TFFMI = trunk fat-free mass index; LFMI $=$ limb fat mass index; LFFMI $=$ limb fat-free mass index; $\Delta=$ Akaike information criterion score reduction relative to the baseline model; $R^{2}=$ measure of explained variation; $C=$ concordance. 
The profiles for mortality risk as a function of both FMI and FFMI (Figure 2c), as well as TFFMI, LFMI, and LFFMI (not shown) were very similar to each other and to that for BMI (Figure 2a), consistent with their very high positive correlations. They all featured increased risk at low values, and lowest risk at near- and above-mean values. TFMI showed somewhat different behavior, with increased risk at values both substantially below and substantially above the population mean (Figure 2d).
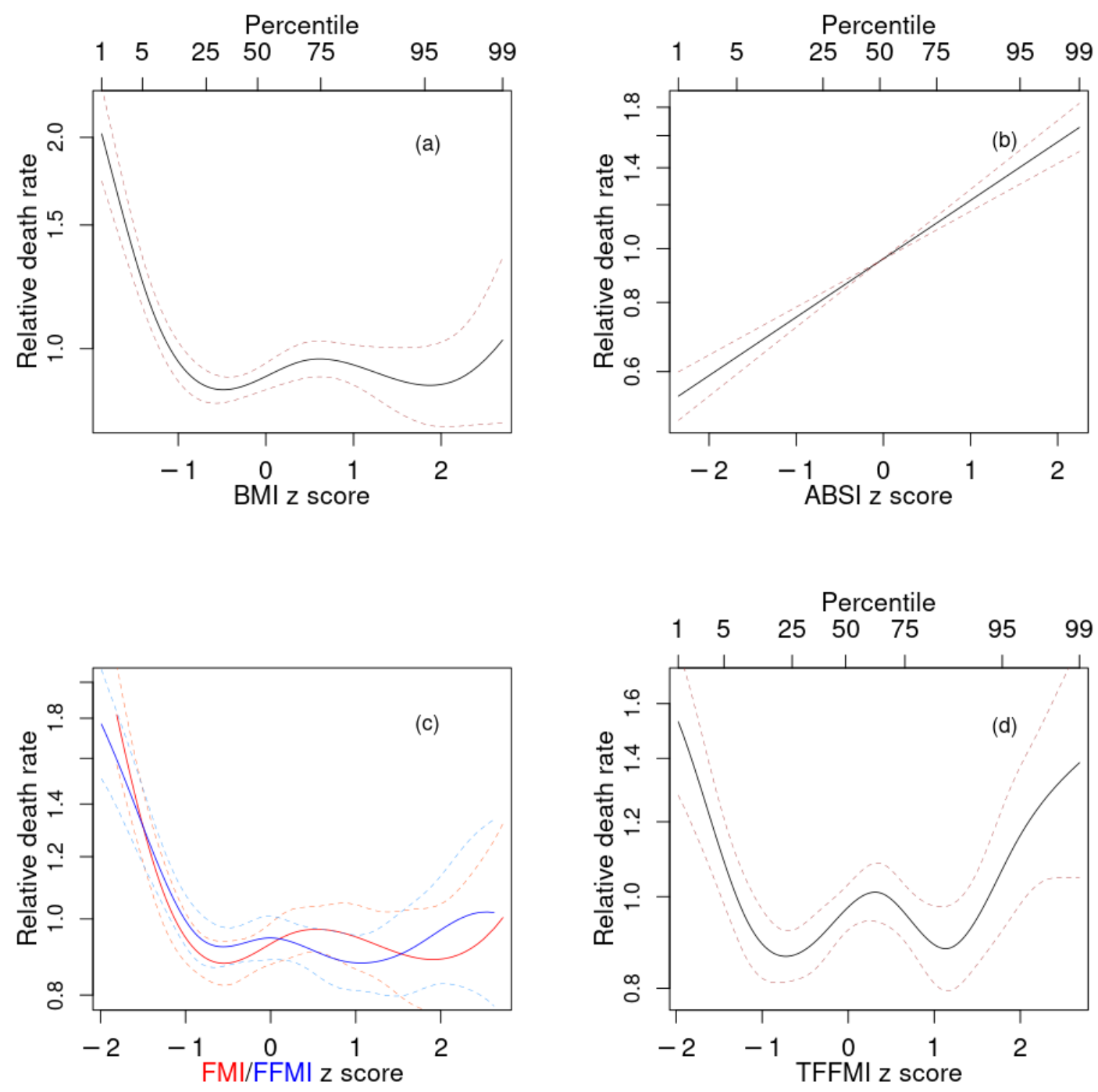

Figure 2. Estimated mortality hazard ratios in NHANES 1999-2006 as nonlinear (penalized spline) functions of (a) BMI, (b) ABSI, (c) FMI (red) and FFMI (blue), (d) TFFMI. Dashed lines indicate 95\% confidence intervals. NHANES = National Health and Nutrition Examination Survey; BMI = body mass index; ABSI = a body shape index; FMI = fat mass index; FFMI $=$ fat-free mass index; TFFMI = trunk fat-free mass index.

Given that ABSI outperformed all the body composition indices as a linear predictor, we next investigated whether adding adjusted body composition indices to a model that also contained ABSI and BMI as predictors resulted in improved prediction over only using BMI and ABSI. These results are shown in Table 4 and Figure 3. As found in previous studies, the combination of BMI and ABSI results in a powerful predictive model for mortality. Their statistical independence helps explain why the increases in $\Delta, R^{2}, C$ in the combined model over baseline were very similar to the sum of the incremental increases for the models with just BMI and just ABSI. Adding body composition indices to this model resulted in changes in $\Delta$ that were either not statistically significant (for FMI and LFMI), 
suggesting that these indices offered no usable mortality prediction improvement over simple anthropometrics, or only marginally statistically significant (for FFMI and TFMI). The two exceptions were TFFMI and LFFMI, which both substantially improved mortality prediction when added to BMI and ABSI. Above-average trunk lean mass (high adjusted TFFMI) was associated with increased mortality hazard, as was below-average limb lean mass (low adjusted LFFMI) (Figure 3). Because adjusted TFFMI and LFFMI bore a positive correlation, these opposite tendencies were better expressed when both were added to the prediction model simultaneously, leading to the combined model (last line in Table 4) showing even more improvement than the sum of that incrementally found in the models that included TFFMI and LFFMI individually.

Table 4. The association of each body composition measure with mortality hazard when considered alongside simple anthropometrics.

\begin{tabular}{cccc}
\hline Predictor & $\boldsymbol{\Delta}$ & $\boldsymbol{R}^{\mathbf{2}}$ & $\boldsymbol{C}$ \\
\hline BMI + ABSI & 195.2 & 0.088 & 0.615 \\
+FMI & 200.6 & 0.096 & 0.618 \\
+FFMI & 207.5 & 0.097 & 0.620 \\
+TFMI & 202.0 & 0.094 & 0.618 \\
+TFFMI & 255.6 & 0.110 & 0.627 \\
+LFMI & 200.9 & 0.097 & 0.620 \\
+LFFMI & 222.4 & 0.100 & 0.619 \\
+TFFMI + LFFMI & 317.6 & 0.130 & 0.635 \\
\hline
\end{tabular}

As in Table 3, but for models including both BMI and ABSI as predictors, and with the body composition indices adjusted to remove correlation with BMI and ABSI. NHANES $=$ National Health and Nutrition Examination Survey; $\mathrm{BMI}=$ body mass index; $\mathrm{WC}=$ waist circumference; $\mathrm{ABSI}=$ a body shape index; $\mathrm{FMI}=$ fat mass index FFMI $=$ fat-free mass index; TFMI $=$ trunk fat mass index; TFFMI $=$ trunk fat-free mass index LFMI $=$ limb fat mass index; LFFMI = limb fat-free mass index; $\Delta=$ Akaike information criterion score reduction relative to the baseline model; $R^{2}=$ measure of explained variation; $C=$ concordance.
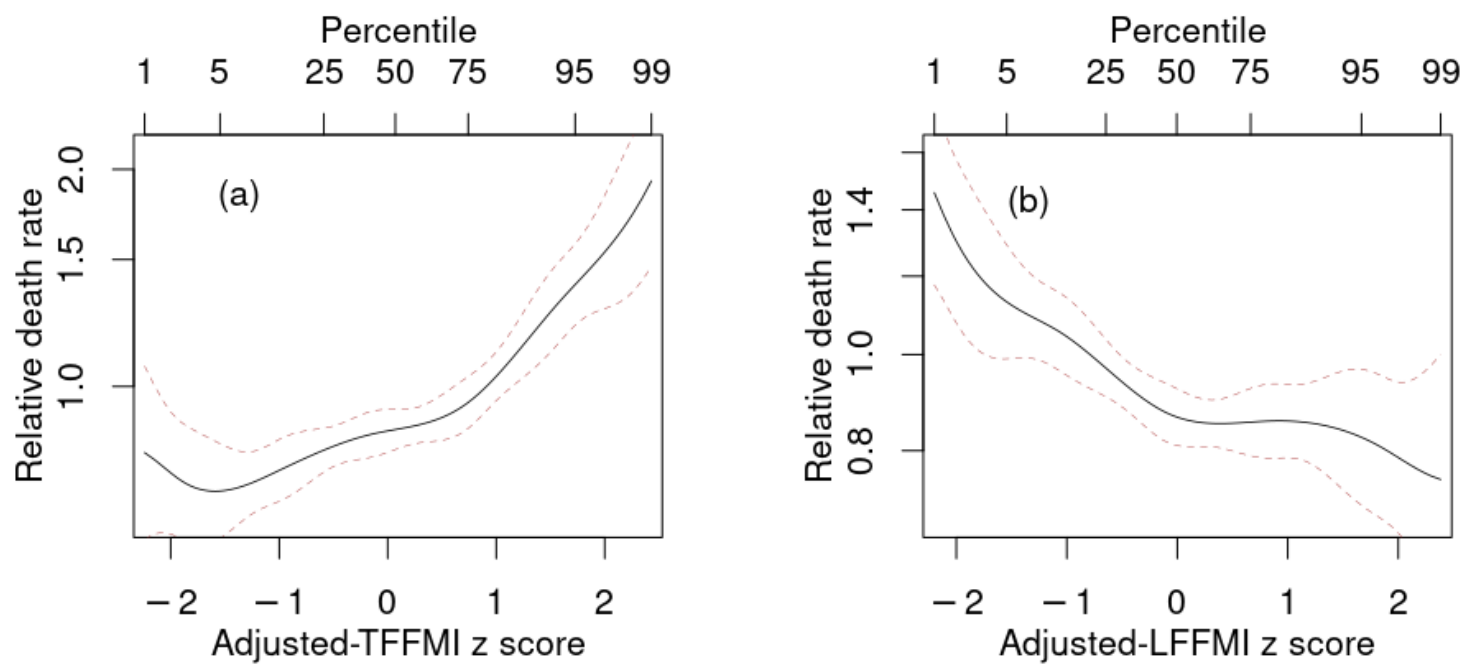

Figure 3. Estimated mortality hazard ratios in NHANES 1999-2006 as nonlinear (penalized spline) functions of adjusted (a) TFFMI, (b) LFFMI in models that also include as predictors BMI and ABSI. Dashed lines indicate 95\% confidence intervals. NHANES = National Health and Nutrition Examination Survey; BMI = body mass index; ABSI = a body shape index; FMI = fat mass index; TFFMI = trunk fat-free mass index; LFFMI = limb fat-free mass index.

\section{Discussion}

DEXA body composition has been rated the gold standard for reasons of precision and low radiation exposure for inferring composition across the three tissue types (bone, lean, fat) from total body or regional (trunk, limbs) areal projections [8]. Over the last 30 years, DEXA densitometers have become a part of routine screening, assessment and monitoring 
of osteoporosis. Many of these same instruments can perform total body scanning and thus provide widespread but unrealized access to body composition measurements. Factors for underutilization have included lack of insurance coverage and normative values. The latter concern has been successfully addressed, largely by the NHANES population data. However, only DEXA bone densitometry is a covered service and performed routinely. Moreover, the low utilization may be most attributable to the failure to establish the clinical utility of DEXA body composition for diagnostic or risk assessment [42].

$\mathrm{BMI}$ and ABSI are allometric anthropometric indices that respectively correct body weight for height and waist circumference for BMI. ABSI was derived to meet allometric criteria of statistical independence from height and BMI [43,44]. In this study, we extended the adjustment of DEXA mass measurements beyond height and BMI to include ABSI. In fact, we found that a power-law dependence on height, BMI, and ABSI can estimate the whole-body DEXA body composition to rather high accuracy, comparable to that reported for other methods for measuring fat mass such as bioelectrical impedance analysis [45]. Anthropometric corrected DEXA body composition values will be independent of BMI and ABSI and may therefore better identify mortality associations with body composition that cannot be inferred from BMI and ABSI alone.

While body size and body composition are conceptually distinct, they are also closely connected. Obesity, as categorized by BMI, is usually associated with relatively more fat tissue, particularly in the trunk, as compared to lean tissue. WC is mostly predictable from height, weight, age, and sex [46], but variability in standardized WC, expressible using ABSI, has considerable diagnostic value, and also correlates with body composition, with higher ABSI associated with more trunk fat and less limb lean tissue. The ratio of height to WC has been used as a proxy for relative fat mass, with threshold values for elevated mortality risk determined using NHANES data [15].

After adjusting for BMI and ABSI, both DEXA measured total fat mass and trunk fat mass showed little correlation with mortality risk. This may reflect the acknowledged inability of conventional DEXA to isolate the most harmful fat depots, such as abdominal visceral fat, as compared to other trunk depots such as superficial subcutaneous fat that are not particularly harmful [7,47-49]. Our finding is consistent with [14], who found that in NHANES 1999-2006 trunk fat percentage was not significantly associated with mortality risk, although it was associated with cardiovascular disease mortality.

On the other hand, above-average adjusted trunk fat-free mass was associated with higher mortality risk, a linkage that, to our knowledge, has not been previously reported for DEXA body composition. One potential mechanism is that excess trunk lean tissue may tend to correspond to enlarged internal organs (organomegaly). For example, enlarged hearts correlate to worse prognosis of patients with cardiac disease [50]. In patients with autosomal dominant polycystic kidney disease, enlarged livers and kidneys indicated a much higher risk of malnutrition [51]. In newly diagnosed patients with symptomatic Waldenstrom macroglobulinemia, enlarged livers and spleens were associated with worse survival [52]. Mice with targeted expression of an Igf2 transgene in smooth muscle cells showed enlarged hearts and shortened lifespan, which was discussed as potentially relevant to the progression of human cardiac diseases [53].

While adjusted trunk fat-free mass was associated with higher mortality, adjusted limb fat-free mass was associated with lower mortality. This is consistent with low muscle mass being a risk factor for death and disability. About $75 \%$ of skeletal muscle mass is in the limbs, and therefore limb measurements using DEXA "can be considered as the reference standard for measuring muscle mass" [54]. Mortality associations using total body composition will be confounded as total body lean tissue includes visceral organs, body fluids and soft tissue calcifications and is only about $50 \%$ muscle [54].

Our findings with regard to trunk and limb fat-free mass could also be explored in relation to the capacity-load model of disease risk [55]. In this model, metabolic capacity is lower for people with low birthweight or childhood stunting, as evinced by less limb muscle mass and smaller internal organs. Metabolic load is imposed by stressors such as 
unhealthy food and exposure to pathogens and toxic pollutants. Lower metabolic capacity reduces the ability to tolerate metabolic load and hence increases vulnerability to disease in later life.

One limitation of our study is that obese people were disproportionately likely not to have valid DEXA scans, which would have led them to be excluded from our analyses. The limitation of DEXA scanners in imaging people with obesity [10] has been mitigated in recent years with newer detectors and software, along with half-body scans for those too large to be completely scanned [5]. To apply the body composition normals and risk profiles inferred from NHANES also requires attention to the properties of different DEXA machines and software.

Another limitation of the present work is that we considered associations in the entire NHANES population with valid data and did not study whether they vary between subgroups defined by factors such as sex, age, or ethnicity. Gene variants associated with high ABSI, for example, were found to differ between women and men [56]. Follow-up work could address this, although inference for subgroups will be limited by smaller sample sizes.

Sarcopenia has come to complement abdominal obesity as a recognized marker of ill health, especially in the elderly, and has been extensively studied [57-59]. Most studies involving anthropometrics or body composition modalities focus on the most conceptually associated measurements. Appendicular muscle (limb lean) mass is used in the definition of sarcopenia, while trunk fat mass is considered an indicator of abdominal obesity and visceral fat. In both NHANES and in the South Korean population, ABSI was found to be a good indicator of the sarcopenia criterion of DEXA-measured low limb lean mass among people with above-threshold waist circumference [60]. However, functional testing of strength and mobility is likely superior to muscle mass for assessing morbidity and mortality attributable to sarcopenia $[61,62]$.

\section{Conclusions}

We report that DEXA-derived whole-body composition (fat or fat-free mass indices), both before and after anthropometric adjustment, adds little skill to mortality prediction compared to anthropometrics alone. For regional DEXA body composition, limb lean and trunk lean do seem to improve on just BMI and ABSI. Thus, based on the present analysis from NHANES data, mortality prediction using DEXA body composition indices may offer little advantage for mortality prediction over basic anthropometrics. WWAnthropometricsadjusted DEXA, however, suggests added value from some body regions. The association between adjusted limb fat-free mass and mortality is consistent with the well-known association of sarcopenia with low appendicular muscle mass. The predictive value of adjusted trunk fat-free mass is a novel finding and perhaps reflects the adverse mortality implication of organomegaly.

Author Contributions: Conceptualization, methodology, validation, investigation, writing (all drafts), administration: all authors. Software and visualization: N.Y.K. Both authors have read and agreed to the published version of the manuscript.

Funding: This research received no external funding.

Institutional Review Board Statement: Not applicable.

Informed Consent Statement: Not applicable.

Data Availability Statement: The public-use NHANES data analyzed in this study are available at https:/ / www.cdc.gov/nchs/nhanes/index.htm, accessed on 1 June 2021.

Conflicts of Interest: The authors declare no conflict of interest. 


\section{References}

1. Ji, M.; Zhang, S.; An, R. Effectiveness of A Body Shape Index (ABSI) in predicting chronic diseases and mortality: A systematic review and meta-analysis. Obes. Rev. 2018, 19, 737-759. [CrossRef]

2. Christakoudi, S.; Tsilidis, K.K.; Muller, D.C.; Freisling, H.; Weiderpass, E.; Overvad, K.; Söderberg, S.; Häggström, C.; Pischon, T.; Dahm, C.C.; et al. A Body Shape Index (ABSI) achieves better mortality risk stratification than alternative indices of abdominal obesity: Results from a large European cohort. Sci. Rep. 2020, 10, 14541. [CrossRef]

3. Snijder, M.; van Dam, R.; Visser, M.; Seidell, J. What aspects of body fat are particularly hazardous and how do we measure them? Int. J. Epidemiol. 2006, 35, 83. [CrossRef]

4. Ahima, R.S.; Lazar, M.A. The health risk of obesity-better metrics imperative. Science 2013, 341, 856-858. [CrossRef] [PubMed]

5. Lee, D.H.; Giovannucci, E.L. Body composition and mortality in the general population: A review of epidemiologic studies. Exp. Biol. Med. 2018, 243, 1275-1285. [CrossRef] [PubMed]

6. Hulkoti, V.S.; Acharya, S.; Shukla, S.; Partapa, S.K.; Gupte, Y. In search of an ideal obesity assessment tool: Is body mass index reliable enough? J. Evol. Med. Dent. Sci. 2020, 9, 2556-2560. [CrossRef]

7. Frank, A.P.; de Santos, R.S.; Palmer, B.F.; Clegg, D.J. Determinants of body fat distribution in humans may provide insight about obesity-related health risks. J. Lipid Res. 2019, 60, 1710-1719. [CrossRef] [PubMed]

8. Shepherd, J.A.; Ng, B.K.; Sommer, M.J.; Heymsfield, S.B. Body composition by DXA. Bone 2017, 104, 101-105. [CrossRef] [PubMed]

9. Ponti, F.; Santoro, A.; Mercatelli, D.; Gasperini, C.; Conte, M.; Martucci, M.; Sangiorgi, L.; Franceschi, C.; Bazzocchi, A. Aging and imaging assessment of body composition: From fat to facts. Front. Endocrinol. 2020, 10, 861. [CrossRef] [PubMed]

10. Laskey, M. Dual-energy X-ray absorptiometry and body composition. Nutrition 1996, 12, 45-51. [CrossRef]

11. Pietrobelli, A.; Formica, C.; Wang, Z.; Heymsfield, S.B. Dual-energy X-ray absorptiometry body composition model: Review of physical concepts. Am. J. Physiol. Endocrinol. Metab. 1996, 271, E941-E951. [CrossRef]

12. Kelly, T.L.; Wilson, K.E.; Heymsfield, S.B. Dual energy X-ray absorptiometry body composition reference values from NHANES PLoS ONE 2009, 4, e7038. [CrossRef]

13. Hinton, B.J.; Fan, B.; Ng, B.K.; Shepherd, J.A. Dual energy X-ray absorptiometry body composition reference values of limbs and trunk from NHANES 1999-2004 with additional visualization methods. PLoS ONE 2017, 12, e0174180. [CrossRef] [PubMed]

14. Zong, G.; Zhang, Z.; Yang, Q.; Wu, H.; Hu, F.B.; Sun, Q. Total and regional adiposity measured by dual-energy X-ray absorptiometry and mortality in NHANES 1999-2006. Obesity 2016, 24, 2414-2421. [CrossRef]

15. Woolcott, O.O.; Bergman, R.N. Defining cutoffs to diagnose obesity using the relative fat mass (RFM): Association with mortality in NHANES 1999-2014. Int. J. Obes. 2020, 44, 1301-1310. [CrossRef] [PubMed]

16. Sedlmeier, A.M.; Baumeister, S.E.; Weber, A.; Fischer, B.; Thorand, B.; Ittermann, T.; Dörr, M.; Felix, S.B.; Völzke, H.; Peters, A.; et al. Relation of body fat mass and fat-free mass to total mortality: results from 7 prospective cohort studies. Am. J. Clin. Nutr. 2021, 113, 639-646. [CrossRef]

17. Wilson, J.P.; Kanaya, A.M.; Fan, B.; Shepherd, J.A. Ratio of trunk to leg volume as a new body shape metric for diabetes and mortality. PLoS ONE 2013, 8, e68716. [CrossRef]

18. Abramowitz, M.K.; Hall, C.B.; Amodu, A.; Sharma, D.; Androga, L.; Hawkins, M. Muscle mass, BMI, and mortality among adults in the United States: A population-based cohort study. PLoS ONE 2018, 13, e0194697. [CrossRef]

19. Van Aller, C.; Lara, J.; Stephan, B.C.; Donini, L.M.; Heymsfield, S.; Katzmarzyk, P.T.; Wells, J.C.; Prado, C.M.; Siervo, M. Sarcopenic obesity and overall mortality: Results from the application of novel models of body composition phenotypes to the National Health and Nutrition Examination Survey 1999-2004. Clin. Nutr. 2019, 38, 264-270. [CrossRef] [PubMed]

20. Zhang, X.; Xie, X.; Dou, Q.; Liu, C.; Zhang, W.; Yang, Y.; Deng, R.; Cheng, A.S.K. Association of sarcopenic obesity with the risk of all-cause mortality among adults over a broad range of different settings: A updated meta-analysis. BMC Geriatr. 2019, 19, 183. [CrossRef] [PubMed]

21. Biolo, G.; Di Girolamo, F.G.; Breglia, A.; Chiuc, M.; Baglio, V.; Vinci, P.; Toigo, G.; Lucchin, L.; Jurdana, M.; Mazzucco, S.; et al. Inverse relationship between "a body shape index" (ABSI) and fat-free mass in women and men: Insights into mechanisms of sarcopenic obesity. Clin. Nutr. 2015, 34, 323-327. [CrossRef]

22. Dhana, K.; Koolhas, C.; Schoufour, J.; Rivadeneira, F.; Hofman, A.; Kavousi, M.; Franco, O.H. Association of anthropometric measures with fat and fat-free mass in the elderly: The Rotterdam study. Maturitas 2016, 88, 96-100. [CrossRef]

23. Krakauer, N.Y.; Krakauer, J.C. An Anthropometric Risk Index based on combining height, weight, waist, and hip measurements. J. Obes. 2016, 2016, 8094275. [CrossRef]

24. Chung, W.; Park, J.H.; Chung, H.S.; Yu, J.M.; Kim, D.S.; Moon, S. Utility of the Z-score of log-transformed A Body Shape Index (LBSIZ) in the assessment for sarcopenic obesity and cardiovascular disease risk in the United States. Sci. Rep. 2019, 9, 9292. [CrossRef]

25. NHANES. National Health and Nutrition Examination Survey Technical Documentation for the 1999-2004 Dual Energy X-ray Absorptiometry (DXA) Multiple Imputation Data Files; Technical Report; National Center for Health Statistics: 2008. Available online: https://wwwn.cdc.gov/nchs/data/nhanes/dxa/dxa_techdoc.pdf (accessed on 8 June 2021)

26. Keys, A.; Fidanza, F.; Karvonen, M.J.; Kimura, N.; Taylor, H.L. Indices of relative weight and obesity. J. Chronic Dis. 1972, 25, 329-343. [CrossRef] 
27. Krakauer, N.Y.; Krakauer, J.C. A new body shape index predicts mortality hazard independently of body mass index. PLoS ONE 2012, 7, e39504. [CrossRef] [PubMed]

28. VanItallie, T.B.; Yang, M.U.; Heymsfield, S.B.; Funk, R.C.; Boileau, R.A. Height-normalized indices of the body's fat-free mass and fat mass: potentially useful indicators of nutritional status. Am. J. Clin. Nutr. 1990, 52, 953-959. [CrossRef]

29. Schutz, Y.; Kyle, U.; Pichard, C. Fat-free mass index and fat mass index percentiles in Caucasians aged 18-98 y. Int. J. Obes. 2002, 26, 953-960. [CrossRef] [PubMed]

30. Cox, D.R. Partial likelihood. Biometrika 1975, 62, 269-276. [CrossRef]

31. Krakauer, N.Y.; Krakauer, J.C. Anthropometrics, metabolic syndrome, and mortality hazard. J. Obes. 2018, $2018,9241904$. [CrossRef] [PubMed]

32. Krakauer, N.Y.; Krakauer, J.C. Dynamic association of mortality hazard with body shape. PLoS ONE 2014, 9, e88793. [CrossRef] [PubMed]

33. Krakauer, N.Y.; Krakauer, J.C. Association of body shape index (ABSI) with hand grip strength. Int. J. Environ. Res. Public Health 2020, 17, 6797. [CrossRef] [PubMed]

34. Burnham, K.P.; Anderson, D.R. Multimodel inference: Understanding AIC and BIC in model selection. Sociol. Methods Res. 2004, 33, 261-304. [CrossRef]

35. Royston, P. Explained variation for survival models. Stata J. 2006, 6, 83-96. [CrossRef]

36. Therneau, T.M.; Grambsch, P.M. Modeling Survival Data: Extending the Cox Model; Springer: New York, NY, USA, 2000.

37. Heagerty, P.J.; Lumley, T.; Pepe, M.S. Time-dependent ROC curves for censored survival data and a diagnostic marker. Biometrics 2000, 56, 337-344. [CrossRef]

38. Pantoja-Galicia, N.; Okereke, O.I.; Blacker, D.; Betensky, R.A. Concordance measures and time-dependent ROC methods. Biostat. Epidemiol. 2021, 1-18. [CrossRef]

39. Hurvich, C.M.; Simonoff, J.S.; Tsai, C.L. Smoothing parameter selection in nonparametric regression using an improved Akaike information criterion. J. R. Stat. Soc. 1998, 60B, 271-293. [CrossRef]

40. R Core Team. R: A Language and Environment for Statistical Computing; R Foundation for Statistical Computing: Vienna, Austria, 2021.

41. Therneau, T.M. A Package for Survival Analysis in R; R Package Version 3.2-7; R Foundation for Statistical Computing: Vienna, Austria, 2020.

42. Krakauer, J.C.; Franklin, B.; Kleerekoper, M.; Karlsson, M.; Levine, J.A. Body composition profiles derived from dual-energy X-ray absorptiometry total body scan and mortality. Prev. Cardiol. 2004, 7, 109-115. [CrossRef]

43. Krakauer, N.Y.; Krakauer, J.C. Untangling waist circumference and hip circumference from body mass index with a body shape index, hip index, and anthropometric risk indicator. Metab. Syndr. Relat. Disord. 2018, 16, 160-165. [CrossRef]

44. Krakauer, N.Y.; Krakauer, J.C. The new anthropometrics and abdominal obesity: A body shape index, hip index, and anthropometric risk index. In Nutrition in the Prevention and Treatment of Abdominal Obesity, 2nd ed.; Watson, R.R., Ed.; Academic Press: Cambridge, MA, USA, 2019; Chapter 2, pp. 19-27. [CrossRef]

45. Achamrah, N.; Colange, G.; Delay, J.; Rimbert, A.; Folope, V.; Petit, A.; Grigioni, S.; Déchelotte, P.; Coëffier, M. Comparison of body composition assessment by DXA and BIA according to the body mass index: A retrospective study on 3655 measures. PLoS ONE 2018, 13, e0200465. [CrossRef]

46. Zhou, W.; Eckler, S.; Barszczyk, A.; Waese-Perlman, A.; Wang, Y.; Gu, X.; Feng, Z.P.; Peng, Y.; Lee, K. Waist circumference prediction for epidemiological research using gradient boosted trees. BMC Med. Res. Methodol. 2021, 21, 47. [CrossRef]

47. Porter, S.A.; Massaro, J.M.; Hoffmann, U.; Vasan, R.S.; O’Donnel, C.J.; Fox, C.S. Abdominal Subcutaneous Adipose Tissue: A Protective Fat Depot? Diabetes Care 2009, 32, 1068-1075. [CrossRef]

48. Dulloo, A.G.; Jacquet, J.; Solinas, G.; Montani, J.P.; Schutz, Y. Body composition phenotypes in pathways to obesity and the metabolic syndrome. Int. J. Obes. 2010, 34, S4-S17. [CrossRef] [PubMed]

49. Goossens, G.H. The Metabolic Phenotype in Obesity: Fat Mass, Body Fat Distribution, and Adipose Tissue Function. Obes. Facts 2017, 10, 207-215. [CrossRef]

50. Justin, M.; Zaman, S.; Sanders, J.; Crook, A.M.; Feder, G.; Shipley, M.; Timmis, A.; Hemingway, H. Cardiothoracic ratio within the "normal" range independently predicts mortality in patients undergoing coronary angiography. Heart 2007, 93, 491-494. [CrossRef] [PubMed]

51. Ryu, H.; Kim, H.; Park, H.C.; Kim, H.; Cho, E.J.; Lee, K.B.; Chung, W.; Oh, K.H.; Cho, J.Y.; Hwang, Y.H.; et al. Total kidney and liver volume is a major risk factor for malnutrition in ambulatory patients with autosomal dominant polycystic kidney disease. BMC Nephrol. 2017, 18, 22. [CrossRef] [PubMed]

52. Ghobrial, I.M.; Fonseca, R.; Gertz, M.A.; Plevak, M.F.; Larson, D.R.; Therneau, T.M.; Wolf, R.C.; Hoffmann, R.J.; Lust, J.A.; Witzig, T.E.; et al. Prognostic model for disease-specific and overall mortality in newly diagnosed symptomatic patients with Waldenstrom macroglobulinaemia. Br. J. Haematol. 2006, 133, 158-164. [CrossRef]

53. Zaina, S.; Pettersson, L.; Thomsen, A.B.; Chai, C.M.; Qi, Z.; Thyberg, J.; Nilsson, J. Shortened life span, bradycardia, and hypotension in mice with targeted expression of an Igf2 transgene in smooth muscle cells. Endocrinology 2003, 144, 2695-2703. [CrossRef]

54. Buckinx, F.; Landi, F.; Cesari, M.; Fielding, R.A.; Visser, M.; Engelke, K.; Maggi, S.; Dennison, E.; Al-Daghri, N.M.; Allepaerts, S.; et al. Pitfalls in the measurement of muscle mass: A need for a reference standard. J. Cachexia Sarcopenia Muscle 2018, 9, 269-278. [CrossRef] 
55. Wells, J.C.K. The capacity-load model of non-communicable disease risk: Understanding the effects of child malnutrition, ethnicity and the social determinants of health. Eur. J. Clin. Nutr. 2018, 72, 688-697. [CrossRef]

56. Christakoudi, S.; Evangelou, E.; Riboli, E.; Tsilidis, K.K. GWAS of allometric body-shape indices in UK Biobank identifies loci suggesting associations with morphogenesis, organogenesis, adrenal cell renewal and cancer. Sci. Rep. 2021, 11, 10688. [CrossRef] [PubMed]

57. Cruz-Jentoft, A.J.; Sayer, A.A. Sarcopenia. Lancet 2019, 393, 2636-2646. [CrossRef]

58. Farmer, R.E.; Mathur, R.; Schmidt, A.F.; Bhaskaran, K.; Fatemifar, G.; Eastwood, S.V.; Finan, C.; Denaxas, S.; Smeeth, L.; Chaturvedi, N. Associations between measures of sarcopenic obesity and risk of cardiovascular disease and mortality: A cohort study and Mendelian randomization analysis using the UK Biobank. J. Am. Heart Assoc. 2019, 8, e011638. [CrossRef]

59. Zeng, Q.; Wang, L.; Dong, S.; Zha, X.; Ran, L.; Li, Y.; Chen, S.; Gao, J.; Li, S.; Lu, Y.; et al. CT-derived abdominal adiposity: Distributions and better predictive ability than BMI in a nationwide study of 59,429 adults in China. Metabolism 2021, 115, 154456. [CrossRef] [PubMed]

60. Moon, S.; Kim, Y.J.; Yu, J.M.; Kang, J.G.; Chung, H.S. Z-score of the log-transformed A Body Shape Index predicts low muscle mass in population with abdominal obesity: The U.S. and Korea National Health and Nutrition Examination Survey. PLoS ONE 2020, 15, e0242557. [CrossRef]

61. Newman, A.B.; Kupelian, V.; Visser, M.; Simonsick, E.M.; Goodpaster, B.H.; Kritchevsky, S.B.; Tylavsky, F.A.; Rubin, S.M.; Harris, T.B. Strength, but not muscle mass, is associated with mortality in the Health, Aging and Body Composition Study cohort. J. Gerontol. Ser. A Biol. Sci. Med. Sci. 2006, 61, 72-77. [CrossRef]

62. Cruz-Jentoft, A.J.; Bahat, G.; Bauer, J.; Boirie, Y.; Bruyère, O.; Cederholm, T.; Cooper, C.; Landi, F.; Rolland, Y.; Sayer, A.A.; et al. Sarcopenia: Revised European consensus on definition and diagnosis. Age Ageing 2018, 48, 16-31. [CrossRef] 